\title{
Power Control by Interference Prediction for Broadband Wireless Packet Networks*
}

\author{
Kin K. Leung \\ AT\&T Labs, Room 5A-1D35 \\ 200 South Laurel Ave \\ Middletown, NJ 07748 \\ Phone: +1 732-420-9041 \\ Email: kkleung@ research.att.com \\ September 22, 1999 \\ January 8, 2001 (Revised)
}

\begin{abstract}
A Kalman-filter method for power control is proposed for broadband, packet-switched TDMA wireless networks. By exploiting the temporal correlation of co-channel interference, a Kalman filter is used to predict future interference power. Based on the predicted interference and estimated path gain between the transmitter and receiver, transmission power is determined to achieve a desired signal-to-interference-plus-noise ratio (SINR). A condition to ensure power stability in the packet-switched environment is established and proven for a special case of the Kalman-filter method. The condition generalizes the existing one for a fixed path-gain matrix, as for circuit-switched networks.

Performance results reveal that the Kalman-filter method for power control provides a significant performance improvement. Specifically, when messages consist of 10 packets on average, the 90th and 95th percentile of the SINR by the new method are 3.79 and $5.46 \mathrm{~dB}$ above those when no power control is in use, and lie just 0.96 and $1.14 \mathrm{~dB}$ below the upper-bound performance of the optimal power control, respectively, in a system with 4-sector cells and an interleaved frequency assignment of a reuse factor of $2 / 8$. In addition, the new method performs noticeably better than the delta-modulation method and a simple scheme that uses the last measurement as predicted interference power. In an example of 8-PSK modulation and average message length of 20 packets, the SINR performance gain by the new method improves the network throughput by about $150 \%$ and $70 \%$, relative to no power control and the simple scheme, respectively.
\end{abstract}

* Part of this paper was presented at IEEE INFOCOM'99, New York, NY, March 1999. 


\section{INTRODUCTION}

There is a growing demand for broadband wireless networks as work-at-home, telecommuting and Internet access have become very popular. Given the proliferation of Internet Protocol (IP) networks, it is important to design broadband wireless networks that support transport of IP packets. Towards this goal, it is natural to allow terminals and base stations to send data continuously until the whole data message is transmitted, as on typical wired IP networks such as Ethernet.

Dynamic transmission power control has been widely studied and practiced to manage interference in cellular radio networks; see e.g., [Z92a], [FM93], [CS94], [EKBNS96], [RZ98] and [UY98]. Specifically, power control has been shown to be a useful technique to improve performance and capacity of time-division-multiple-access (TDMA) wireless networks. In addition to performance improvement, power control is actually essential in solving the near-far problem in code-division-multiple-access (CDMA) networks. In this paper, we focus on broadband packet-switched TDMA networks with data rates up to several megabits per second.

Existing power control algorithms can be categorized into two classes: signal-based and signal-to-interference-ratio (SIR) based power control. Signal-based power control [W93] [HWJ97] adjusts the transmission power based on the received signal strength. In contrast, SIRbased control [Z92a], [Z92b], [FM93], [GVG94], [CS94] changes the power according to the ratio of signal and co-channel interference (possibly plus noise) power levels. (Since only co-channel interference is considered here, it is simply referred to as interference in the following.) It has been shown that SIR-based power control outperforms signal-based control.

Many SIR-based power control algorithms implicitly assume that calls have relatively long holding time and they use the last SIR measurement to adjust power iteratively. However, they may not be efficient for packet-switching networks due to the burstiness of data packets, coupled with irregular transmission schedule in these networks. Thus, we need an appropriate power control for packet-switched TDMA networks, and this is the topic of this paper.

To help illustrate our ideas for wireless packet networks, let us assume that time is divided into slots. Let each data message be divided into a number of packets, each of which can be transmitted in one time slot. As in typical IP networks, the message length (in terms of the number of packets) varies randomly from message to message. Despite such randomness, the networks allow multiple, contiguous time slots to be used by the same terminal or base station for transmitting a message. As a consequence, the interference at a given receiver is correlated from 
one time slot to the next. We observe that such temporal correlation for the interference becomes strong quickly when the message length increases from one. For this reason, based on the interference measurements in previous slots, one can apply appropriate methods to predict the interference power to be received at a terminal or base station in the next slot. Based on the predicted interference and estimated path gain between the transmitter and receiver, the transmission power in the next time slot can be determined to achieve the desired performance in terms of signal-to-interference-plus-noise ratio (SINR). These are the key ideas behind the power control algorithm proposed in the following.

In particular, we propose to use a Kalman filter [BH97] [H96] to predict the interference power, thus our algorithm is referred to as the Kalman-filter method. The advantage of the Kalman filter is that it is simple, due to its recursive structure and robust over a wide range of parameters, and it possibly provides an optimal estimate in the sense of minimum mean square error. Kalman filters have been applied successfully to many systems [BH97]. As for wireless networks, [DJM96] proposes using a Kalman filter for call admission in CDMA networks. We report here that Kalman filtering is also useful for power control in TDMA networks.

The organization of the rest of this paper is as follows. In section 2, the Kalman-filter method for power control is presented. The stability of power control in wireless packet networks is analyzed in Section 3. Then, in Section 4, we use simulation techniques to study the performance of the new method. Finally, we present our conclusions in Section 5.

\section{A KALMAN-FILTER METHOD FOR POWER CONTROL}

Although the Kalman-filter power control is applicable to both the uplink (from terminal to base station) and the downlink (from base station to terminal), we focus on the uplink here.

\subsection{System Assumptions}

1. Consider a cellular radio network where time is divided into slots. Let each data message be divided into a number of packets, each of which can be sent in one time slot. The network allows multiple, contiguous time slots to be used by the same transmitter for sending a message, thus producing temporal correlation for interference.

2. The path gain (i.e., the path loss plus shadow fading) between a terminal and its base station can be estimated accurately by measurements; for example, by use of the control channel for handoff purposes in GSM system [R96]. This assumption is reasonable, especially for the case where the path gain does not change much over time when the terminal is moving 
at a very slow speed or is stationary.

3. The medium-access control (MAC) protocol in use allows at most one terminal in each sector or cell to send data at a time; that is, no data contention occurs within the same sector or cell. In addition, the base station knows which terminal is scheduled to transmit at different times. (E.g., typical polling and reservation schemes meet both requirements.) When a terminal transmits, it can send packets in multiple time slots contiguously.

4. Due to the large volume of data involved, base stations do not exchange control information among themselves on a per packet basis in real time. Thus, it is extremely difficult to estimate how much interference one transmission causes to others.

5. Interference power in each time slot can be measured quickly, but probably with errors at each base station. The interference power is equal to the difference between the total received power and the power of the desired signal, where the latter can be measured by filtering based on the training symbols for the signal. In fact, making such measurements can be challenging, especially when time duration is short; see e.g., [AS95] and [AMY98].

\subsection{Interference Prediction by Kalman Filter}

We use a Kalman filter to predict the future interference-plus-noise power. Let $I(n)$ be the actual interference-plus-noise power in $\mathrm{dBm}$ received at a given base station in time slot $n$. In other words, $I(n)$ is the "process state" to be estimated by the Kalman filter. Assume that the noise power, which depends on the channel bandwidth, is given and fixed. For brevity, unless stated otherwise, the interference plus thermal noise is simply referred to as interference in the following. The dynamics of the interference power is described by

$$
I(n)=I(n-1)+F(n)
$$

where $F(n)$ represents the fluctuation of interference power as terminals start new transmissions and/or adjust their transmission power in the time slot. In the terminology of Kalman filtering, $F(n)$ is the "process noise." In essence, the interference power is modeled as a Brownian-motion process [BH97] in (1). Let $Z(n)$ be the measured interference power in $\mathrm{dBm}$ for slot $n$. Then,

$$
Z(n)=I(n)+E(n)
$$

where $E(n)$ is the "measurement noise" (e.g., due to receiver noise). Eq.(1) and (2) are commonly referred to as the signal generation model. By the Kalman filter theory [BH97], the time and measurement update equations for the interference power are: 


$$
\begin{aligned}
& \tilde{I}(n+1)=\hat{I}(n) \\
& \tilde{P}(n+1)=\hat{P}(n)+Q(n) \\
& K(n)=\tilde{P}(n)[\tilde{P}(n)+R(n)]^{-1} \\
& \hat{I}(n)=\tilde{I}(n)+K(n)[Z(n)-\tilde{I}(n)] \\
& \hat{P}(n)=[1-K(n)] \tilde{P}(n)
\end{aligned}
$$

where $\tilde{I}(n)$ and $\hat{I}(n)$ are the a priori and a posteriori estimates of $I(n), \tilde{P}(n)$ and $\hat{P}(n)$ are the $a$ priori and a posteriori estimate-error variances, $K(n)$ is the Kalman gain, and $Q(n)$ and $R(n)$ are the variances for the process noise $F(n)$ and measurement noise $E(n)$, respectively.

Clearly, $Q(n)$ and $R(n)$ need to be estimated as input for (4) and (5). As an initial attempt, we estimate $Q(n)$ based on interference measurements in the last $W$ slots as follows:

$$
\begin{aligned}
& \bar{Z}(n)=\frac{1}{W} \sum_{i=n-W+1}^{n} Z(i) \\
& Q(n) \approx \frac{1}{W-1} \sum_{i=n-W+1}^{n}[Z(i)-\bar{Z}(n)]^{2} .
\end{aligned}
$$

This approximation of $Q(n)$ is obtained based on the following reasoning. First, $\bar{Z}(n)$ given by (8) is the average measured interference power (noise included) over the sliding window $W$. We observe that as $I(n)$ and $E(n)$ in (2) are typically independent, the variance of $Z(n)$ over the sliding window, as given by the right-hand side of (9), actually represents the sum of the variances of the interference power $I(n)$ and the measurement noise $E(n)$. Since the fluctuation of $I(n)$ can reach as much as tens of decibels in the packet-switched networks, which is much higher than typical measurement errors, the variance of $Z(n)$ approximately equals the variance of $I(n)$. In turn, as shown in (9), the approximate variance of $I(n)$ is used as an estimate of the variance $Q(n)$ of the process noise $F(n)$, which is the change in interference power from one time slot to the next according to (1). This last step of approximation is appropriate because $Q(n)$ is proportional to the fluctuation of $I(n)$. We note that the sliding window in (8) and (9) is used in order to capture the non-stationarity of interference. Evidently, $W$ should be chosen big enough (e.g., $\geq 10$ ) so that if the measurement errors $E(n)$ in (2) have zero mean (which is typical), (8) can yield an unbiased estimate of average interference power.

Actually, $R(n)$ depends on the error characteristics of interference measurements at the receiver, and is typically uncorrelated with $Q(n)$. In practice, $R(n)$ can be determined by, for example, measuring the "received" power on a known, idle channel. Thus, the variance of the 
"received" power over a time window is an estimate of $R(n)$. To illustrate the potential performance gain of the Kalman-filter method without considering details of the measurement error characteristics and procedure here, we assume for simplicity that $R(n)$ is given by

$$
R(n)=\zeta Q(n)
$$

where $\zeta$ is a given constant between 0 and 1. The choice of $R(n)$ in (10) as an illustrative example is reasonable for our study here because, as mentioned above, $Q(n)$ in (9) includes the variance of measurement noise. We remark that determining the best estimates for $Q(n)$ and $R(n)$ represents an area for further study. Nevertheless, we show in the following that the power-control method using the above estimates can yield significant performance gain. Further gain may be achievable with an enhanced estimate of $Q(n)$ and an actual measurement of $R(n)$.

For each slot $n$, the interference measurements are input to (8) to (10) for estimating $Q(n)$ and $R(n)$. Using these values and the current measurement, (5) to (7) yield the Kalman gain and the a posteriori estimates for $I(n)$ and $P(n)$, respectively. The a priori estimates for the next time

slot are given by (3) and (4). In particular, $\tilde{I}(n+1)$ in (3) is used as the predicted interference power in slot $n+1$ for power control as described below. Before discussing the determination of transmission power, we emphasize that although the Kalman filter in (3) to (7) is presented as a tool for interference prediction, it also provides smoothing effects as the measurement noise (error) is smoothed out by the nature of filtering.

\subsection{Determination of Transmission Power}

Let $\gamma$ be the target SINR, $p(n)$ the transmission power and $g(n)$ the path gain from the transmitting terminal to the base station for slot $n$, respectively. While $I(n)$ and $\tilde{I}(n)$ represent the actual and predicted interference power in $\mathrm{dBm}$, we use $i(n)$ and $\tilde{i}(n)$ to denote the respective values in $\mathrm{mW}$. By Assumption 2, the base station can estimate $g(n)$ accurately. Based on this and the predicted interference $\tilde{I}(n)$ in (3), the base station instructs (via a downlink channel) the terminal to transmit in slot $n$ with power

$$
p(n)=\gamma \frac{\tilde{i}(n)}{g(n)} .
$$

The goal of this setting of transmission power is to choose just enough power to achieve the target SINR $\gamma$, thus minimizing interference to others without degrading one's link quality. In fact, different targets can be used in (11) for different terminals, depending on their link quality [LW00]. Nevertheless, we assume all terminals have an identical target SINR here. 
When power is selected by (11), the receiving SINR $\gamma(n)$ at the base station is

$$
\gamma(n)=\frac{p(n) g(n)}{i(n)}=\gamma \frac{\tilde{i}(n)}{i(n)},
$$

where $i(n)$ is the actual interference power in $\mathrm{mW}$ for slot $n$. It is clear from (12) that when the interference prediction by the Kalman filter is accurate (i.e., $\tilde{i}(n) \approx i(n)$ ), the target SINR is

achieved. Even when $\tilde{i}(n)$ does not predict $i(n)$ exactly, the method also helps in reducing the spread of $\gamma(n)$, as long as $\tilde{i}(n)$ and $i(n)$ are positively correlated.

\subsection{Steps for the Kalman-Filter Method}

The Kalman-filter method for power control is summarized as:

a. For each time slot $n$, each base station measures the interference power for the time slot.

b. The interference measurements are used as input to the Kalman filter in (3) to (10) to predict the interference power $\tilde{I}(n+1)$ (or equivalently, $\tilde{i}(n+1))$ in slot $n+1$.

c. Based on the MAC protocol in use (which satisfies Assumption 3), the base station tracks the path gain $g(n+1)$, and selects the transmission power by (11) to meet a given target SINR for the terminal that transmits in slot $n+1$.

d. The power level $p(n+1)$ is forwarded via the downlink to the terminal for actual transmission.

\section{STABILITY OF POWER CONTROL IN WIRELESS PACKET NETWORKS}

Instead of considering the general case of the Kalman-filter method, we consider a simple power-control scheme where the transmission power for slot $n$ is determined by

$$
p(n)=\gamma \frac{z(n-1)}{g(n)} .
$$

where $z(n-1)$ is the measured interference power in $\mathrm{mW}$ for slot $n-1$; that is, $z(n-1)$ is the linear-scale equivalent of $Z(n-1)$ in $\mathrm{dB}$ in (2). Actually, (13) is a special case of the Kalmanfilter method. Specifically, if the measurement noise is constant (i.e., $R(n)=0$ for all $n$ ), we have $K(n)=1$ and $\tilde{I}(n+1)=Z(n)=I(n)+E(n)$, according to (2) to (7). In other words, the measured interference power in slot $n-1$ is used as an estimate of that same quantity in slot $n$. In this case, (11) becomes (13). It is noteworthy that (13) also corresponds to the power control with $\beta=1$ in [FM93] and that in [GVG94] when receiver noise is ignored. 
To continue, let the network have $M$ co-channel sectors and $J$ terminals in each sector, where exactly one terminal is scheduled to transmit in each sector in a time slot. For $i, j=1$ to $M$, we use $g_{i j}(n)$ to denote the path gain (i.e., the path loss plus shadowing) from a terminal that transmits in slot $n$ in sector $j$ to the base station receiver of sector $i$. For each slot $n$, let $\mathbf{G}(n)$ be the path-gain matrix $\left[g_{i j}(n-1) / g_{i i}(n)\right]_{\mathrm{MxM}}$ with elements in the diagonal replaced by zeros. As in [Z92a], we assume that $\mathbf{G}(n)$ for all $n$ has a full rank with probability one. Let $\lambda_{i}(n)$ and $\mathbf{u}_{i}(n)$ for $i=1$ to $M$ be the eigenvalues and associated eigenvectors of $\mathbf{G}(n)$ with $\left|\lambda_{1}(n)\right| \geq\left|\lambda_{2}(n)\right| \geq \cdots \geq\left|\lambda_{M}(n)\right|$ To study power stability, it is appropriate to consider the $l_{\infty}$ norm of a vector $\mathbf{x}=\left(x_{1}, x_{2}, \cdots, x_{M}\right)^{T}$, denoted by $\|\mathbf{x}\|_{\infty} \equiv \max _{1 \leq i \leq M}\left|x_{i}\right|$. To avoid extreme situations, we also assume that all eignvectors associated with $\mathbf{G}(n)$ have finite $l_{\infty}$ norm; i.e., $\left\|\mathbf{u}_{i}(n)\right\|_{\infty}<\infty$ for all $i$ and $n$, with probability one.

With $M$ sectors in the system and $J$ terminals in each sector, different combinations of terminals can transmit in a time slot, which result in $J^{M}$ possible path-gain matrices applicable to every slot. Let these matrices be denoted by $\mathbf{G}_{i}$ for $i=1$ to $J^{M}$. Further, let $\beta_{i}$ be the largest eigenvalue for each $\mathbf{G}_{i}$. Clearly, as a result of the packet switching, $\mathbf{G}(n)$ is equal to one of the $\mathbf{G}_{i}$ 's for each slot $n$, depending on which terminals are actually transmitting in the slot. We assume that the choices of transmitting terminals are made randomly and independent of system conditions. In addition, for $k=1$ to $J^{M}$, let $a_{k}$ be the long-term (i.e., over a sufficiently large number of time slots) fraction of occurrence of $\mathbf{G}(n)=\mathbf{G}_{k}$, for $n=1,2, \ldots, \infty$. Clearly, $\sum_{k=1}^{J^{M}} a_{k}=1$.

Let the power vector be $\mathbf{p}(n)=\left(p_{1}(n), \ldots, p_{M}(n)\right)^{T}$ where $p_{i}(n)$ is the power for the terminal of sector $i$ transmitting in slot $n$. We note that the standard notion of "convergence" of the power vector may not apply well in wireless packet networks because different sets of terminals can be transmitting in consecutive time slots. Rather, our main concern is whether the transmission powers of all terminals remain finite in the steady state. Thus, we define a power-control algorithm to be stable, if $\lim _{n \rightarrow \infty} p_{i}(n)<\infty$ for all $i$. That is, if $\lim _{n \rightarrow \infty}\|\mathbf{p}(n)\|_{\infty}<\infty$.

\subsection{Stability for Interference-Limited Case}

Without the receiver (thermal) noise, $z(n-1)=i(n-1)=\sum_{j \neq i} g_{i j}(n-1) p_{j}(n-1)$. Based on this, the power control in (13) for the whole system can be expressed in a matrix form:

$$
\mathbf{p}(n)=\gamma \mathbf{G}(n) \mathbf{p}(n-1)
$$

Theorem 1: With $\|\mathbf{p}(0)\|_{\infty}<\infty, \lim _{n \rightarrow \infty}\|\mathbf{p}(n)\|_{\infty}<\infty$ if and only if 


$$
\gamma \prod_{k=1}^{J^{M}} \beta_{k}^{a_{k}}<1
$$

where for $k=1$ to $J^{M}, a_{k}$ is the long-term fraction of occurrence of $\mathbf{G}(n)=\mathbf{G}_{k}$ for $n=1,2, \ldots, \infty$.

Proof: As given by (14), the power vector for slot $n, \mathbf{p}(n)$, is determined as a function of the power vector for the previous slot, $\mathbf{p}(n-1)$. However, our approach in the proof is to express a given power vector $\mathbf{p}(n)$ as a sum of eignvectors associated with the path-gain matrix $\mathbf{G}(n+1)$ for slot $n+1$. Specifically, since $\mathbf{G}(1)$ has a full rank, its eignvectors $\mathbf{u}_{j}(1)$, for $j=1$ to $M$, are linearly independent. Thus, for a given $\mathbf{p}(0)$, we can express

$$
\mathbf{p}(0)=\sum_{i_{1}=1}^{M} \mathbf{u}_{i_{1}}(1)
$$

Substituting (16) into (14) and using the property of matrix eigenvectors and eigenvalues that $\mathbf{G}(1) \mathbf{u}_{i}(1)=\lambda_{i}(1) \mathbf{u}_{i}(1)$ for all $i$, we have

$$
\mathbf{p}(1)=\gamma \mathbf{G}(1) \sum_{i_{1}=1}^{M} \mathbf{u}_{i_{1}}(1)=\gamma \lambda_{1}(1) \sum_{i_{1}=1}^{M} \frac{\lambda_{i_{1}}(1)}{\lambda_{1}(1)} \mathbf{u}_{i_{1}}(1) .
$$

Similarly, $\mathbf{u}_{i_{1}}$ (1) in (17) can now be represented by a sum of linearly independent eigenvectors $\mathbf{u}_{i_{1}, i_{2}}$ (2)'s of $\mathbf{G}(2)$ for slot 2. That is, for $i_{1}=1$ to $M$,

$$
\mathbf{u}_{i_{1}}(1)=\sum_{i_{2}=1}^{M} \mathbf{u}_{i_{1}, i_{2}}(2)
$$

Then, recursively expanding (14) yields

$$
\mathbf{p}(n)=\gamma^{n} \prod_{i=1}^{n} \lambda_{1}(i) \sum_{i_{1}=1}^{M} \sum_{i_{2}=1}^{M} \cdots \sum_{i_{n}=1}^{M} \frac{\lambda_{i_{1}}(1)}{\lambda_{1}(1)} \cdot \frac{\lambda_{i_{2}}(2)}{\lambda_{1}(2)} \cdots \frac{\lambda_{i_{n}}(n)}{\lambda_{1}(n)} \mathbf{u}_{i_{1}, i_{2}, \ldots, i_{n}}(n),
$$

where, for $k=2$ to $n, \mathbf{u}_{i_{1}, i_{2}, \ldots, i_{k-1}}(k-1)=\sum_{i_{k}=1}^{M} \mathbf{u}_{i_{1}, i_{2}, \ldots, i_{k}}(k)$, and the latter are linearly independent eigenvectors of $\mathbf{G}(k)$ for slot $k$. Using the fact that $\frac{\left|\lambda_{i_{k}}(k)\right|}{\left|\lambda_{1}(k)\right|} \leq 1$ for all $k$, we take the $l_{\infty}$ norm of both sides of (19). Further, since $\left\|\mathbf{u}_{i_{1}, i_{2}, \ldots, i_{n}}(n)\right\|_{\infty}<\infty$ for all $n$, let $K$ be a finite upper bound of these $l_{\infty}$ norms. Finally, taking $n \rightarrow \infty$, we obtain from (19) that 


$$
\begin{aligned}
\lim _{n \rightarrow \infty}\|\mathbf{p}(n)\|_{\infty} & \leq \lim _{n \rightarrow \infty} \gamma^{n} \prod_{i=1}^{n} \lambda_{1}(i) \sum_{i_{1}=1}^{M} \sum_{i_{2}=1}^{M} \cdots \sum_{i_{n}=1}^{M}\left\|\mathbf{u}_{i_{1}, i_{2}, \ldots, i_{n}}(n)\right\|_{\infty} \\
& \left.\leq \lim _{n \rightarrow \infty} \gamma^{n} \prod_{i=1}^{n} \lambda_{1}(i) M K n=M K \lim _{n \rightarrow \infty} n \mid \gamma \prod_{k=1}^{J^{M}} \beta_{k}^{a_{k}}\right\rceil^{n} .
\end{aligned}
$$

where the conversion of the product of the $\lambda_{1}(i)$ 's into that of the $\beta_{k}$ 's is valid due to the following reason. By definition, when $n \rightarrow \infty$ and for each $k=1$ to $J^{M}, n a_{k}$ is the number of occurrence that $\mathbf{G}(i)=\mathbf{G}_{k}$ for $i=1$ to $n$. Given $\mathbf{G}(i)=\mathbf{G}_{k}$, their largest eignvalues equal to each other; that is, $\lambda_{1}(i)=\beta_{k}$. Thus, $\prod_{i=1}^{n} \lambda_{1}(i)=\prod_{k=1}^{J^{M}} \beta_{k}^{n a_{k}}$ when $n \rightarrow \infty$. Applying Theorem 3.20d in [R76], the last limit in (20) converges as $n \rightarrow \infty$ if and only if (15) holds. Thus, $\lim _{n \rightarrow \infty}\|\mathbf{p}(n)\|_{\infty}$ is bounded if and only if (15) is true.

It is worth noting that Theorem 1 is a generalization of the standard condition $\gamma \lambda<1$ (e.g., [Z92a], [GVG94]) for power stability, where $\lambda$ is the maximum eigenvalue of a given fixed path gain matrix, as in circuit-switched networks.

\subsection{A Sufficient Stability Condition in Presence of Receiver Noise}

Suppose that the constant receiver noise power at the base station of sector $i$ is $\eta_{i}$. We use $\eta(n)$ to denote the vector of $\eta_{i} / g_{i i}(n)$, for $i=1$ to $M$ and $n \geq 1$. In parallel to (14), the power control in (13) with receiver noise can be expressed as

$$
\mathbf{p}(n)=\gamma[\mathbf{G}(n) \mathbf{p}(n-1)+\eta(n)]
$$

Before proving that (15) is a sufficient condition for the stability of (21), we define

$$
A_{n} \equiv \gamma^{n-1} \prod_{i=2}^{n} \lambda_{1}(i)(n-1)+\gamma^{n-2} \prod_{i=3}^{n} \lambda_{1}(i)(n-2)+\gamma^{n-3} \prod_{i=4}^{n} \lambda_{1}(i)(n-3)+\cdots+\gamma \lambda_{1}(n)
$$

Lemma 1: If (15) holds, $\lim _{n \rightarrow \infty} A_{n}<\infty$.

Proof: We apply the root test [R76] to show that the sequence $\left\{A_{n}\right\}$ converges. Let

$$
\omega=\limsup _{n \rightarrow \infty}\left[\gamma^{n-1} \prod_{i=2}^{n} \lambda_{1}(i)(n-1)\right]^{1 / n}
$$

For sufficiently large $n, \prod_{i=2}^{n} \lambda_{1}(i)=\prod_{k=1}^{J^{M}} \beta_{k}^{n a_{k}}$, as in (20). Applying this and (15) to (23) yields 


$$
\omega=\gamma \prod_{k=1}^{J^{M}} \beta_{k}^{a_{k}} \limsup _{n \rightarrow \infty}(n-1)^{1 / n}=\gamma \prod_{k=1}^{J^{M}} \beta_{k}^{a_{k}}<1
$$

By the root-test theorem with $\omega<1,\left\{A_{n}\right\}$ converges. Applying Theorem 3.2c in [R76], the convergence implies that $\left\{A_{n}\right\}$ is bounded.

Theorem 2: Assume that $\|\mathbf{p}(0)\|_{\infty}<\infty$ and $\|\eta(n)\|_{\infty}<\infty$ for all $n$. If (15) holds, then $\lim _{n \rightarrow \infty}\|\mathbf{p}(n)\|_{\infty}<\infty$, with $\mathbf{p}(n)$ given by $(21)$.

Proof: Note that $\mathbf{p}(n)$ in (21) is determined as a function of $\mathbf{p}(n-1)$. Similar to Theorem 1, our approach to the proof is to express a given power vector $\mathbf{p}(n)$ as a sum of linearly independent eignvectors associated with the path-gain matrix $\mathbf{G}(n+1)$ for slot $n+1$. Thus, we can express a given $\mathbf{p}(0)$ as

$$
\mathbf{p}(0)=\sum_{i_{1}=1}^{M} \mathbf{u}_{i_{1}}(1)
$$

where the $u_{i_{1}}$ (1)'s are independent eigenvectors of G(1). Substituting (25) into (21) and using the fact that $\mathbf{G}(1) \mathbf{u}_{i}(1)=\lambda_{i}(1) \mathbf{u}_{i}(1)$ for all $i$, we obtain

$$
\mathbf{p}(1)=\gamma\left\lceil\lambda_{1}(1) \sum_{i_{1}=1}^{M} \frac{\lambda_{i_{1}}(1)}{\lambda_{1}(1)} \mathbf{u}_{i_{1}}(1)+\eta(1)\right\rfloor .
$$

Similar to (18), we express $\mathbf{u}_{i_{1}}(1)=\sum_{i_{2}=1}^{M} \mathbf{u}_{i_{1}, i_{2}}(2)$, for each $i_{1}=1$ to $M$, and $\eta(1)=\sum_{i_{2}=1}^{M} \mathbf{v}_{i_{1}, i_{2}}(2)$, where the $\mathbf{u}_{i_{1}, i_{2}}$ (2)'s and $\mathbf{v}_{i_{1}, i_{2}}$ (2)'s are eigenvectors of $\mathbf{G}(2)$. Thus, recursively expanding (21) leads to

$$
\begin{aligned}
\mathbf{p}(n)= & \gamma^{n} \prod_{i=1}^{n} \lambda_{1}(i) \sum_{i_{1}=1}^{M} \cdots \sum_{i_{n}=1}^{M} \frac{\lambda_{i_{1}}(1)}{\lambda_{1}(1)} \frac{\lambda_{i_{2}}(2)}{\lambda_{1}(2)} \cdots \frac{\lambda_{i_{n}}(n)}{\lambda_{1}(n)} \mathbf{u}_{i_{1}, i_{2}, \ldots, i_{n}}(n) \\
& +\gamma^{n} \prod_{i=2}^{n} \lambda_{1}(i) \sum_{i_{2}=1}^{M} \cdots \sum_{i_{n}=1}^{M} \frac{\lambda_{i_{2}}(2)}{\lambda_{1}(2)} \cdots \frac{\lambda_{i_{n}}(n)}{\lambda_{1}(n)} \mathbf{v}_{i_{2}, i_{3}, \ldots, i_{n}}(n) \\
& +\gamma^{n-1} \prod_{i=3}^{n} \lambda_{1}(i) \sum_{i_{3}=1}^{M} \cdots \sum_{i_{n}=1}^{M} \frac{\lambda_{i_{3}}(3)}{\lambda_{1}(3)} \cdots \frac{\lambda_{i_{n}}(n)}{\lambda_{1}(n)} \mathbf{v}_{i_{3}, i_{4}, \ldots, i_{n}}(n)+\cdots \\
& +\gamma^{2} \lambda_{1}(n) \sum_{i_{n}=1}^{M} \frac{\lambda_{i_{n}}(n)}{\lambda_{1}(n)} \mathbf{v}_{i_{n}}(n)+\gamma \eta(n)
\end{aligned}
$$

where, for $k=2$ to $n$ and $k>j, \mathbf{v}_{i_{j}, i_{j+1}, \ldots, i_{k-1}}(k-1)=\sum_{i_{k}=1}^{M} \mathbf{v}_{i_{j}, i_{j+1}, \ldots, i_{k}}(k)$, and the latter are eigenvectors of $\mathbf{G}(k)$. Consider the $l_{\infty}$ norm and the fact that $\left|\lambda_{i}(j) / \lambda_{1}(j)\right| \leq 1$, for all $i$ and $j$, in (27), we have 


$$
\begin{aligned}
\|\mathbf{p}(n)\|_{\infty} \leq & \gamma^{n} \prod_{i=1}^{n} \lambda_{1}(i) \sum_{i_{1}=1}^{M} \cdots \sum_{i_{n}=1}^{M}\left\|\mathbf{u}_{i_{1}, i_{2}, \ldots, i_{n}}(n)\right\|_{\infty}+\gamma^{n} \prod_{i=2}^{n} \lambda_{1}(i) \sum_{i_{2}=1}^{M} \cdots \sum_{i_{n}=1}^{M}\left\|\mathbf{v}_{i_{2}, i_{3}, \ldots, i_{n}}(n)\right\|_{\infty} \\
& +\cdots+\gamma^{2} \lambda_{1}(n) \sum_{i_{n}=1}^{M}\left\|\mathbf{v}_{i_{n}}(n)\right\|_{\infty}+\gamma\|\eta(n)\|_{\infty} .
\end{aligned}
$$

Since $\left\|\mathbf{u}_{i_{1}, i_{2}, \ldots, i_{n}}(n)\right\|_{\infty}<\infty$ and $\left\|\mathbf{v}_{i_{j}, \ldots, i_{n}}(n)\right\|_{\infty}<\infty$, for all $j \geq 2$, let $K$ be a finite upper bound of these $l_{\infty}$ norms. Applying this to (28) yields

$$
\begin{aligned}
\|\mathbf{p}(n)\|_{\infty} \leq & \gamma^{n} \prod_{i=1}^{n} \lambda_{1}(i) M K n+\gamma K M\left|\gamma^{n-1} \prod_{i=2}^{n} \lambda_{1}(i)(n-1)+\gamma^{n-2} \prod_{i=3}^{n} \lambda_{1}(i)(n-2)+\cdots+\gamma \lambda_{1}(n)\right|_{]} \\
& +\gamma\|\eta(n)\|_{\infty}
\end{aligned}
$$

Similar to (20), when (15) holds and $n \rightarrow \infty$, the first term on the RHS of (29) becomes zero. In addition, since the last term is finite, $\|\mathbf{p}(n)\|_{\infty}<\infty$ if the series in brackets is bounded. Indeed, Lemma 1 confirms the latter, given that (15) holds.

Note that Theorem 2 generalizes the stability criterion in [FM93], where the path-gain matrix is assumed to be constant. However, due to analytical difficulty, we are not able to prove that (15) is the necessary stability condition for (21). We also point out that the computation for (15) is very involved because of a huge number of applicable path-gain matrices, as a result of the many possible combinations of transmitters in the packet-switched network. Lastly, the stability criteria for the general case of Kalman-filter power control is an open research issue. Perhaps one possible direction to study it may be to include another "noise" term in (21) to account for the perturbation of power due to the filtering effects. When appropriate criteria are satisfied, the Kalman filter remains stable, which in turn keeps the new "noise" term finite. If this could be shown, the rest of the proof for Theorem 2 could remain applicable.

\section{PERFORMANCE STUDY}

\subsection{Simulation Model}

Given the complexity of the Kalman-filter method, we choose to use simulation techniques to study its effectiveness. We simulate the cell layout and interleaved channel assignment (ICA) [WL00] in Figure 1. A total of 19 cells in the hexagonal layout are considered. Each cell is divided into 4 sectors, each of which is served by a base station antenna located at the center of the cell. The beamwidth of each base station antenna is $60^{\circ}$, while terminals have omni-directional antennas. For simplicity while closely modeling practical antennas, the radiation pattern for the 
base station antenna is assumed to have a parabolic shape; that is, a $3 \mathrm{~dB}$ drop occurs at the beamwidth half angle and any direction beyond a threshold angle in a clockwise or anti-clockwise direction suffers a given, fixed attenuation relative to the gain at the front direction, which is called the front-to-back (FTB) ratio. For the $60^{\circ}$ base station antenna with $20 \mathrm{~dB}$ FTB ratio, this pattern yields a $3 \mathrm{~dB}$ drop at the $30^{\circ}$ angle in the clockwise or anti-clockwise direction from the front direction, the threshold angle is $77.5^{\circ}$ and the antenna has a gain of $7.38 \mathrm{dBi}$ at the front direction. The ICA (static) scheme in Figure 1 allows sectors with the same labels to use the same channel set, thus yielding a frequency reuse factor of 2/8 (i.e., reuse in every 2 cells or 8 sectors). Each sector is populated with 500 randomly placed and uniformly distributed terminals. Each terminal selects the base station that provides the strongest signal power. Terminals are assumed to be stationary throughout the simulation. To provide accurate results, only statistics in the middle cell in Figure 1 are collected and reported below.

Each radio link is characterized by a path-loss model with an exponent of 4 and lognormal shadow fading with a standard deviation of $8 \mathrm{~dB}$. Fast fading is not considered in obtaining the SINR statistics, but is considered when mapping the SINR performance to packet error rate. Cell radius is $1 \mathrm{Km}$ and the path loss at $100 \mathrm{~m}$ from the cell center is $-78 \mathrm{~dB}$ (for a carrier frequency at 2 $\mathrm{GHz}$ ). Thermal noise power is equal to $-110 \mathrm{dBm}$ to consider $1 \mathrm{MHz}$ channel bandwidth and a noise factor of $4 \mathrm{~dB}$.

Message length is assumed to have a discrete form of Pareto distribution, which has been shown to be appropriate for modeling IP traffic [WTSW97]. More precisely, each time a terminal transmits a message, $i$, the number of slots used in the message transmission, is characterized by the following cumulative distribution function:

$$
H_{i}=1-(k / i)^{\alpha} \quad \text { for } i \geq k \in Z^{+} \text {and } \alpha>1
$$

where $k$ and $\alpha$ are given parameters. Then, the probability that a message consists of $i$ packets is given by $\left.\left.h_{i}={ }_{k} / i\right)^{\alpha}-\Upsilon_{k /(i+1)}\right)^{\alpha}$ for $i \geq k \in Z^{+}$and the average message length is

$$
\left.L=k+\sum_{i=k+1}^{\infty} \Upsilon_{k / i}\right)^{\alpha} .
$$

Since the Pareto distribution has an infinite variance if $\alpha \leq 2$, such values should be avoided. Otherwise, our simulation cannot reach a steady state and results will not have statistical significance. Thus, to guarantee finite variance, for a given $L$, we set $k=\lceil L / 2\rceil$ to ensure $\alpha>2$, where $\lceil y\rceil$ is the smallest integer greater than or equal to $y$. Using this $k$ value, $\alpha$ can be solved from (31) for the given $L$. 
To illustrate our ideas without considering details of measurement noise characteristics, $W$ and $\zeta$ for (8) to (10) are set to be 30 and 0.5, respectively. (Actually, our extensive numerical experiments reveal that the Kalman-filter method gives good results for a wide range of $W$ and $\zeta$, which corresponds to drastically different characteristics of measurement noise.) For convenience, our simulation model assumes that terminals in all cells are synchronized at the slot boundary for transmission. Furthermore, unless stated otherwise (see the discussion associated with Figure 5), we assume $100 \%$ traffic load in this study. That is, there are always terminals ready for transmission in co-channel sectors. Thus, after a terminal transmits a message with a random length according to (30), the base station immediately schedules another randomly chosen terminal in the same sector to start a new transmission in the next time slot. At each time slot with a packet transmission in a sector, its base station computes and records the SINR according to the actual signal and interference power. With such statistics collected over a sufficiently long simulation time, the distribution function for the SINR is obtained, including the high percentiles of the SINR. To help us study the dynamic range for the power control schemes, the model first assumes no limit on the actual transmission power in Section 4.2. Then, the power is limited between 0 and $30 \mathrm{dBm}$ in subsequent subsections. The model assumes that interference power in one time slot can be measured and used to determine the transmission power for the next slot.

\subsection{Performance Gain of the Kalman-Filter Method}

Figure 2 compares the SINR performance for the Kalman-filter method with that for no, full and optimal power control. For no power control, transmission power is fixed at $30 \mathrm{dBm}$, while the full power control scheme fully compensates for the path gain between a terminal and its base station (i.e., the combined path loss and shadow fading) such that the received signal strength at the base station is maintained at a fixed level of $-80 \mathrm{dBm}$.

Results for the optimal power control, shown by solid line in Figure 2, are obtained by the method in [GVG94] based on SIR without considering thermal noise. The method assumes precise knowledge of path gain for all combinations of terminals and base stations. Based on the path-gain matrix, the iterative method is executed until convergence to determine the optimal transmission power for each time slot. The transmission power is scaled in each iteration to avoid numerical underflow and overflow. Thus, these results can be viewed as the upper bounds for the actual SINR performance for the system in Figure 1.

As for the Kalman-filter method, we set the SINR target $\gamma$ to be $17 \mathrm{~dB}$ in (11). As shown by the dashed lines in Figure 2, when the message length is 1, the high (e.g., 90th to 99th) percentiles of the SINR for the Kalman-filter method are very close to those for the full and no power control 
scheme. However, the performance for the new method improves rapidly as the average message length $L$ increases. In particular, for $L=10$, the 90th and 95th percentile of the SINR are 3.79 and $5.46 \mathrm{~dB}$ above the respective percentiles for no power control, which represent very significant performance improvement, and lie just 0.96 and $1.14 \mathrm{~dB}$ below the upper-bound performance of the optimal power control, respectively.

Such improvement can best be explained by examining (12). As $L$ increases, the temporal correlation for interference becomes strong, and the interference power $\tilde{i}(n)$ predicted by the Kalman filter is close to the actual value, $i(n)$, thus achieving the SINR target. When possible, the Kalman-filter method is expected to perform even closer to the target when $L$ is increased further. As expected, the probability for SINR exceeding $17 \mathrm{~dB}$, the SINR target, for the Kalman-filter method drops quickly in Figure 2. This implies that good radio links can transmit at a low power, thus further reducing the amount of interference for co-channel sectors. In addition to the results for Pareto-distributed message lengths in Figure 2, we also obtained similar results for geometrically-distributed lengths.

Let us discuss about the selection of $L$ in Figure 2. For a data rate of $1 \mathrm{Mbps}$, a time slot can be chosen to be $0.5 \mathrm{msec}$; that is, each packet contains 500 bits, which is comparable to the length of a TCP/IP acknowledgement (e.g., in web browsing applications) or the size of an ATM cell when the IP is supported by the ATM transport network. With these parameters, $L$ is likely to be greater than 10 for applications such as telecommuting, where text emails can easily contain more than 5,000 bits.

For $L=10$, Figure 3 illustrates how the SINR performance for the Kalman-filter method improves when the SINR target, $\gamma$ in (11), increases from 13 to $20 \mathrm{~dB}$. As the target increases, the performance becomes close to optimum. Although not shown in the figure, analysis in Section 3 has revealed that there exists a certain maximum target for given channel assignment, cell layout and radio parameters, without increasing transmission power to infinity. However, determining the power stability for a given SINR target is involved in the computation. Thus, we choose to determine the maximum stable (achievable) SINR target for the Kalman-filter method by simulation experiments. For the system in Figure 1, it is found that when the SINR target is greater than $20 \mathrm{~dB}$, the simulation simply did not reach a steady state and the transmission power was set to be extremely high. For $L=10$, Figure 4 shows the distribution of transmission power for selecting 15, 17 and $20 \mathrm{~dB}$ as the SINR target. We note that although a target of $20 \mathrm{~dB}$ is still stable, it requires excessive transmission power. At the other extreme, for $15 \mathrm{~dB}$ as the target SINR, terminals do not fully utilize their typical, maximum transmission power of $30 \mathrm{dBm}$. From 
these results, a maximum target of $17 \mathrm{~dB}$ can be supported in practice.

\subsection{Performance Comparison With Other Methods}

To have meaningful comparisons between the Kalman method and other methods, the transmission power is bounded between 0 and $30 \mathrm{dBm}$ in the rest of this study. The SINR target is set to $17 \mathrm{~dB}$, which is appropriate for the power limits shown in Figure 4.

Table 1 compares the SINR performance of the Kalman method with the delta-modulation method studied in [CS94]. In the delta-modulation method, when a terminal starts a new message transmission, the transmission power for its first packet is set according to the signal path gain so that the receiving signal strength is $-80 \mathrm{dBm}$. For the subsequent packets, the receiving SINR is measured at each time slot. If the SINR is less (greater) than the $17 \mathrm{~dB}$ target, transmission power is increased (decreased) by $1 \mathrm{~dB}$ for the next packet. As indicated above, the power is limited to be between 0 and $30 \mathrm{dBm}$. Table 1 shows that the SINR percentiles improve as message length increases for both methods. However, the Kalman method consistently yields a gain of 1.3 to 4.7 $\mathrm{dB}$ for the SINR percentiles over the delta-modulation method.

Our next step is to assess the performance of the proposed method in the presence of random measurement errors. That is, for a given average relative (absolute) measurement error (say $X \%$ ) and the actual interference $I_{i}$ in $\mathrm{dBm}$ for slot $i$, the interference measurement $Z(i)$, input to $(8)$, is randomly distributed between $I_{i}(1-2 X / 100)$ and $I_{i}(1+2 X / 100) \mathrm{dBm}$. Furthermore, one may wonder how the performance of the Kalman-filter method compares with that of the simple power control in (13). The simple control adjusts the transmission power $p(n)$ for each terminal transmitting in slot $n$ according to (11), with $\tilde{i}(n)$ (which is the predicted interference by the Kalman filter) replaced by $z(n-1)$. This simple scheme is based on the following observation: Despite the fact that the interference power changes in time, it might not change drastically, especially when $L>>1$ and/or the interference comes from many random sources, where the law of large number starts to apply (i.e., to keep the total interference power at its overall average level). As a result, the interference measurement in slot $n-1$, namely $z(n-1)$, can approximate the actual interference power in slot $n$ as well. As pointed out in Section 3, the simple scheme is identical to the Kalman method with zero $R_{n}$. In addition, the simple scheme can also be viewed as a possible implementation of the approach with $\beta=1$ in [FM93], although the latter method does not propose use of interference tracking to adjust power for the first packet of each message transmission.

To illustrate the potential improvement of network capacity by the Kalman-filter method, we consider an example of the packet error rate (PER) (or packet error probability) in Table 2. 
These parameters are adopted from Figure 7 in [E98] for 8-PSK modulation with a data rate of 22.8 Kbps in the Enhanced Data rates for the GSM Evolution (EDGE) system. The throughput and PER performance of the Kalman-filter method and the simple scheme for selected average message lengths with average relative measurement error of $5 \%$ are presented in Table 3 . The throughput is the product of $22.8 \mathrm{Kbps}$ and the associated successful reception probability. For reference, the results for no power control are also given. We first note that when the message length increases, the throughput and PER for both methods improve as the accuracy of the interference prediction improves. Secondly, the PER for no power control is $7.3 \%$, compared to $5.1 \%$ and $2.8 \%$ when $L=20$, for the simple scheme and the Kalman method, respectively. For real-time services such as IP voice, the required PER is a couple of percent [FHCS99]. Thus, the PER for no power control and for the simple scheme will both be inadequate for such services. Even for non-real-time data services, it has been shown [p.13, B98] that a few percent of PER can significantly reduce data throughput at the network protocol layer. Thus, it is desirable to keep the PER satisfactorily low. Without using complicated techniques, one way to improve the PER is to decrease traffic load and thereby reduce interference, as examined in the following.

For a given traffic load $\rho$ per sector and $L$, each sector stays idle for a number of slots after its transmitting terminal finishes a message transmission and before another terminal begins a new transmission. As an example, the idle period is assumed to be geometrically distributed; that is, the idle period lasts for $k$ slots with probability $P_{I}^{k}\left(1-P_{I}\right)$ where $P_{I}=(1-\rho) L / \rho+(1-\rho) L$. Let us use the PER's achieved by the Kalman method with $\rho=1$ and various $L$ values in Table 3 as the required PER's. We perform a binary search on $\rho$ for no power control and also for the simple scheme, so that the reduced traffic load can support the required PER. Figure 5 shows the throughput improvement of the Kalman-filter method relative to no power control and relative to the simple scheme. In particular, to support the required PER of $2.8 \%$, achievable by the Kalman method with $L=20$ with $\rho=1$, the traffic load for no power control and for the simple scheme have to be reduced to 0.4 and 0.59, respectively. As shown in Figure 5, the Kalman method thus provides a relative throughput increase of $150 \%$ and $69.5 \%$ over no power control and the simple scheme, respectively.

Generally, the performance gain of the Kalman-filter method reduces when the control delay increases or message length decreases; see additional results in [L99]. A technique for handling increased control delay and/or decreased message length can be found in [LO0]. 


\section{CONCLUSIONS AND FUTURE WORK}

The Kalman-filter method for power control has been proposed for broadband packetswitched TDMA wireless networks. By observing the temporal correlation of co-channel interference when transmitters can send data packets contiguously, the method uses a Kalman filter to predict future interference power. Based on the predicted interference and estimated path gain between the transmitter and receiver, transmission power is determined to achieve a desired SINR performance. The new technique is simple to implement due to its recursive structure and is robust over a wide range of parameters. A condition to ensure power stability in the packetswitched system is established and proven for a special case of the Kalman-filter method. The condition generalizes that for circuit-switched networks where path-gain matrices are fixed.

Our performance results reveal that the Kalman-filter method for power control provides a significant performance improvement in wireless packet networks. Specifically, for an average message length of 10 packets, the 90th and 95th percentile of the SINR by the new method are 3.79 and $5.46 \mathrm{~dB}$ above those when no power control is in use, and lie just 0.96 and $1.14 \mathrm{~dB}$ below the performance of the optimal SIR-based power control, respectively, in a system of 4-sector cells using the interleaved channel assignment with a frequency reuse factor of $2 / 8$ [WL00]. In addition, the new method performs noticeably better than the delta-modulation method and a simple scheme that uses the last measurement as predicted interference power. In an example of 8-PSK modulation and average message length of 20 packets, the SINR performance gain by the new method improves the network throughput by about $150 \%$ and $70 \%$ relative to no power control and the simple scheme, respectively.

Performance of future wireless networks will depend on the design of dynamic channel assignment (DCA), traffic scheduling, power control, MAC, adaptive antenna, and adaptive modulation/coding for link adaptation. Often, these issues are studied separately. With the proposed power-control algorithm as a basis, we are in the process of developing and studying new designs to consider these issues jointly (e.g., see [LW00]), with a goal of achieving high spectral efficiency and capacity in practical networks.

\section{ACKNOWLEDGMENTS}

Thanks are due to Paul Henry for his comments that helped identify errors in the early results of this work. The author also thanks Justin Chuang and Li-Chun Wang for their discussion, and Martin Eisenberg for his careful reading of this paper. Lastly, thanks are due to the anonymous reviewers for their comments to improve the presentation of this paper. 


\section{REFERENCES}

[AMY98] M. Andersin, N. B. Mandayam and R. D. Yates, "Subspace Based Estimation of the Signal to Interference Ratio for TDMA Cellular Systems," Wireless Networks, Vol. 4, 1998, pp. 241-247.

[AS95] M. D. Austin and G. L. Stüber, "In-Service Signal Quality Estimation for TDMA Cellular Systems," Wireless Personal Communications, Vol. 2, No. 3, 1995, pp. 245-254.

[B98] E. A. Brewer, et al., "A Network Architecture for Heterogeneous Mobile Computing," IEEE Personal Communications, Oct. 1998, pp. 8-24.

[BH97] R. G. Brown and P. Y. C. Hwang, Introduction to Random Signals and Applied Kalman Filtering, 3rd Edition, John Wiley \& Sons, New York (1997).

[CS94] J. C. Chuang and N. R. Sollenberger, "Uplink Power Control for TDMA Portable Radio Channels," IEEE Trans. on Veh. Tech., Vol. 43, No. 1, Feb. 1994, pp. 33-39.

[DJM96] Z. Dziong, M. Jia and P. Mermelstein, "Adaptive Traffic Admission for Integrated Services in CDMA Wireless-Access Networks," IEEE J. on Select. Areas in Commun., Vol. 14, No. 9, Dec. 1996, pp. 1737-1747.

[E98] EDGE - Evaluation of 8-PSK, ETSI SMG2, WPB\#4, Finland, April 1998, Ericsson.

[EKBNS96] Special issue on CDMA Networks, IEEE J. on Select. Areas in Commun., Vol. 14, No. 9, Dec. 1996, A.K. Elhakeem, et. al. (Ed.).

[FHCS99] T. Fingsheidt, T. Hindelang, R. V. Cox, N. Seshradi, "A Study of Voice Transmission Over EDGE Using the IS-641 Speech Coder," contribution 3G.IP/99.07.20.46, July 20, 1999, Telenor Expo, Oslo, Norway.

[FM93] G. J. Foschini and Z. Miljanic, "A Simple Distributed Autonomous Power Control Algorithm and its Convergence," IEEE Trans. on Veh. Tech., Vol. 42, Nov. 1993, pp. 641646.

[GVG94] S. A. Grandhi, R. Vijayan and D. J. Goodman, "Distributed Power Control in Cellular Radio Systems," IEEE Trans. on Commun., Vol. 42, Feb-April 1994, pp. 226-228.

[H96] S. Haykin, Adaptive Filter Theory, 3rd Edition, Prentice Hall, New Jersey (1996). 
[HWJ97] Z. J. Haas, J. H. Winters and D. S. Johnson, "Simulation Results of the Capacity of Cellular Systems," IEEE Trans. on Vech. Tech., Vol. 46, Nov. 1997, pp. 805-817.

[L99] K. K. Leung, "A Kalman-Filter Method for Power Control in Broadband Wireless Networks," Proc. of IEEE Infocom'99, New York, NY, March 1999, pp. 948-956.

[L00] K. K. Leung, "Power Control by Kalman Filter With Error Margin for Wireless IP Networks," Proc. of IEEE WCNC 2000, Chicago, IL, Sept. 2000.

[LW00] K. K. Leung and L. C. Wang, "Controlling QoS by Integrated Power Control and Link Adaptation in Broadband Wireless Networks," European Transactions on Telecommunications, Vol. 11, July/August 2000, pp. 383-394; early results in Proc. IEEE PIMRC'99, Japan, Sept. 1999, pp. 1174-1180.

[R76] W. Rudin, Principles of Mathematical Analysis, 3rd Edition, McGraw-Hill, 1976.

[R96] T. S. Rappaport, Wireless Communications: Principles and Practice, New York: IEEE Press and Prentice Hall, 1996.

[RZ98] Special issue, Wireless Networks 4 (1998) 3, Zvi Rosberg and Jens Zander (Ed.).

[UY98] S. Ulukus and R. D. Yates, "Stochastic Power Control for Cellular Radio Systems," IEEE Trans. on Commun., Vol. 46, June 1998, pp. 784-798.

[W93] J. F. Whitehead, "Signal-level-based Dynamic Power Control for Co-channel Interference Management, Proc. of IEEE Vech. Tech. Conf., Secaucus, NJ, May 1993, pp. 499-502.

[WL00] L. C. Wang and K. K. Leung, "A High-Capacity Wireless Network by Quad-Sector Cell and Interleaved Channel Assignment," IEEE J. on Select. Areas in Commun., Vol. 18, March 2000, pp. 472-480.

[WTSW97] W. Willinger, M. Taqqu, R. Sherman and D. Wilson, "Self-Similarity Through HighVariability: Statistical Analysis of Ethernet LAN Traffic at the Source Level," IEEE/ACM Trans. on Networking, Vol. 5, Feb. 1997, pp. 71-86.

[Z92a] J. Zander, "Performance of Optimum Transmitter Power Control in Cellular Radio Systems," IEEE Trans. on Veh. Tech., Vol. 41, Feb. 1992, pp. 57-62.

[Z92b] J. Zander, "Distributed Cochannel Interference Control in Cellular Radio Systems," IEEE Trans. on Veh. Tech., Vol. 41, Aug. 1992, pp. 305-311. 
Figure 1. A 4-Sector Cell Layout and Interleaved Channel Assignment (ICA)

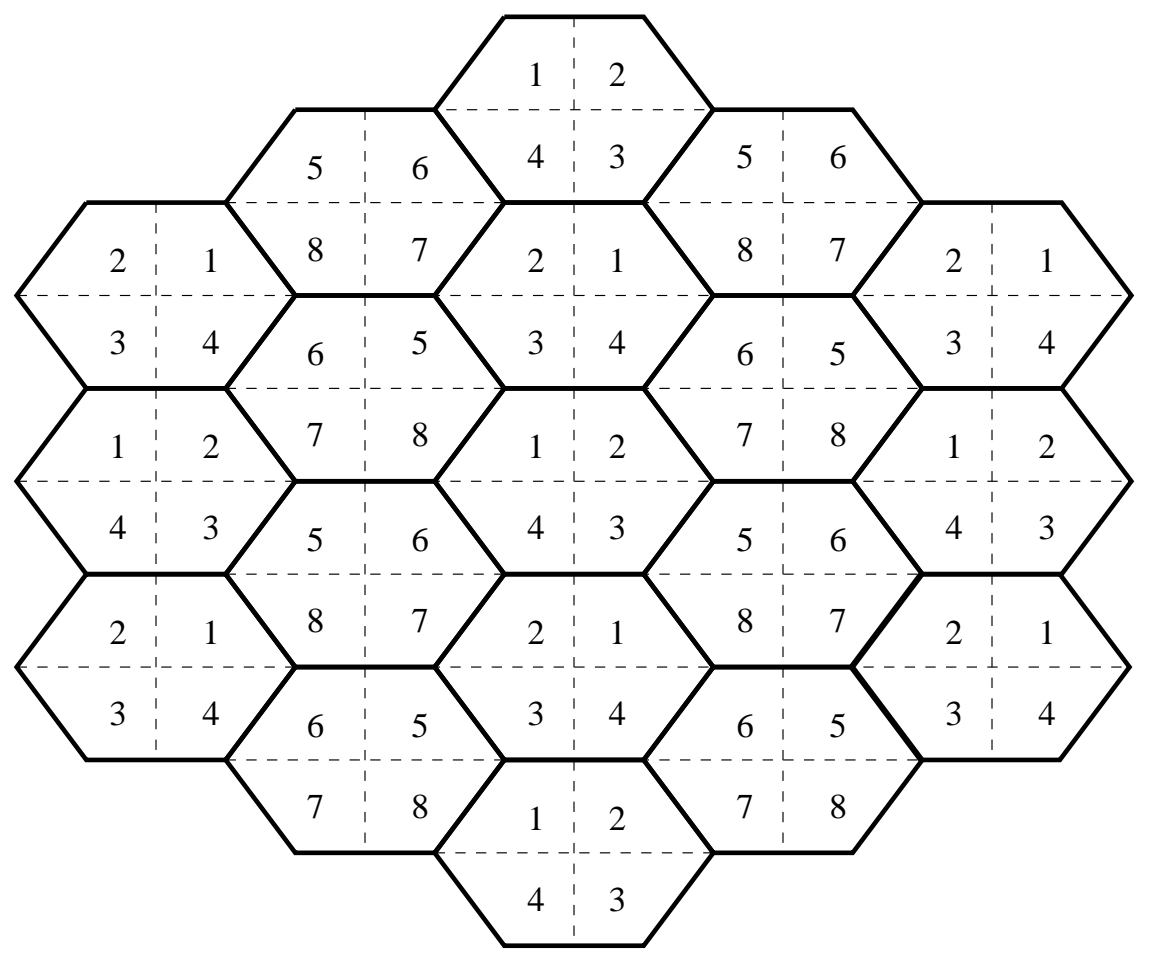


Figure 2. Improvement of SINR Performance

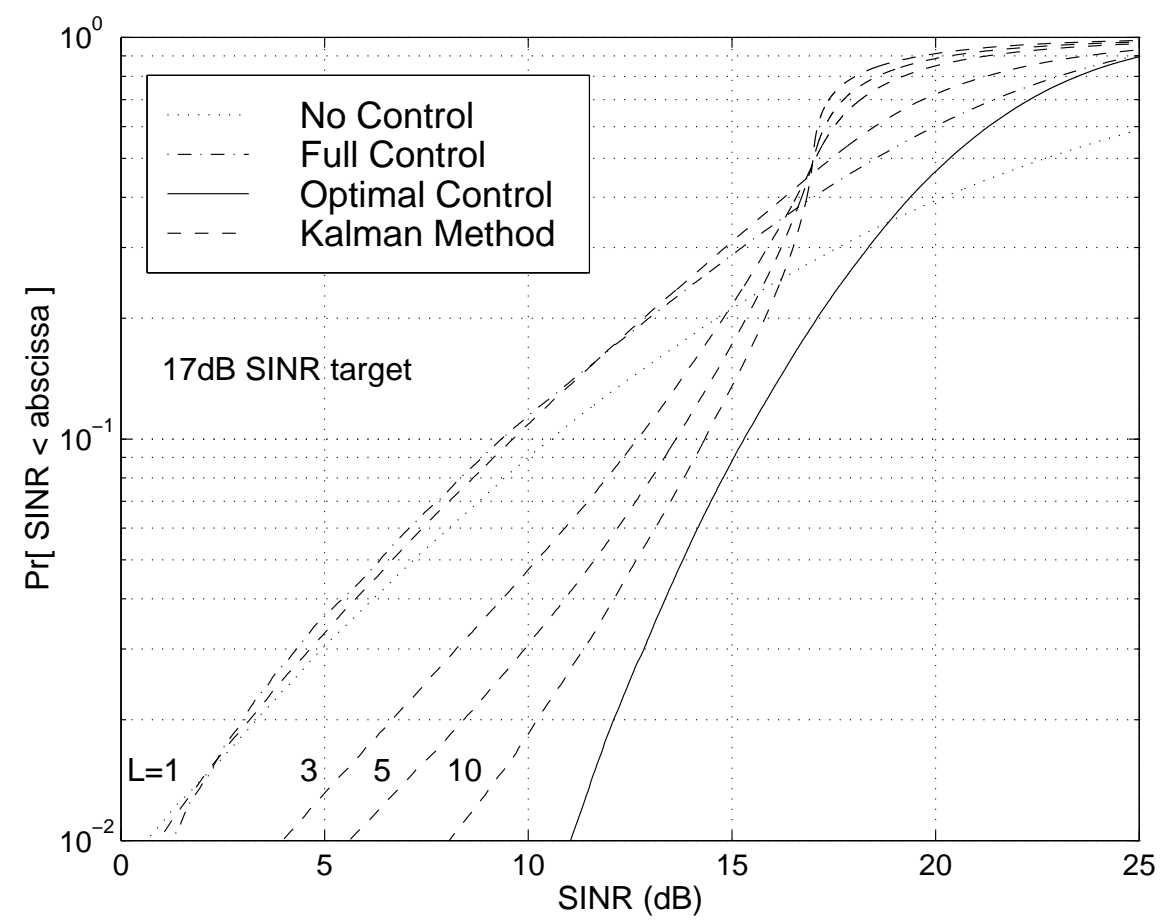

Figure 3. Performance Impacts of SINR Target for the Kalman-Filter Method

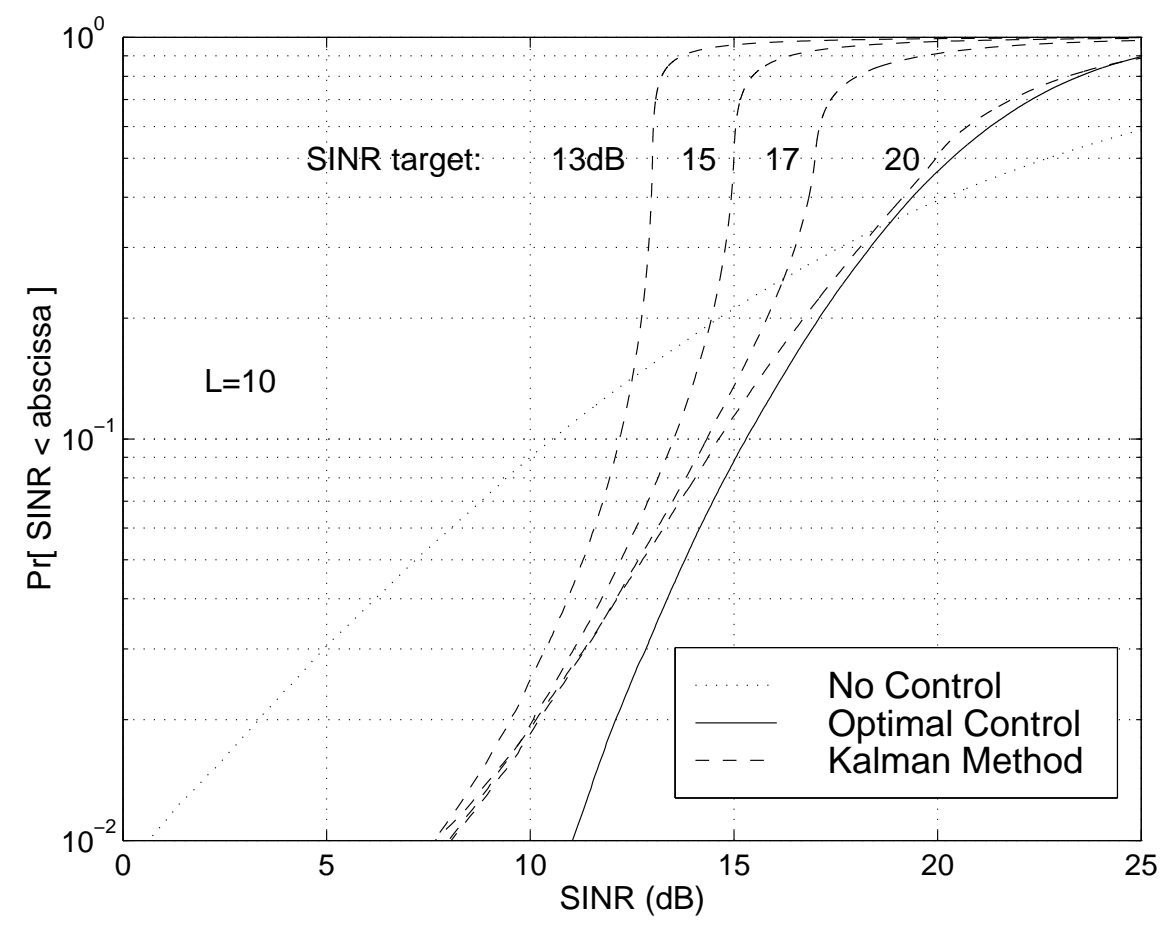


Figure 4. Transmission Power Distribution for the Kalman-Filter Method

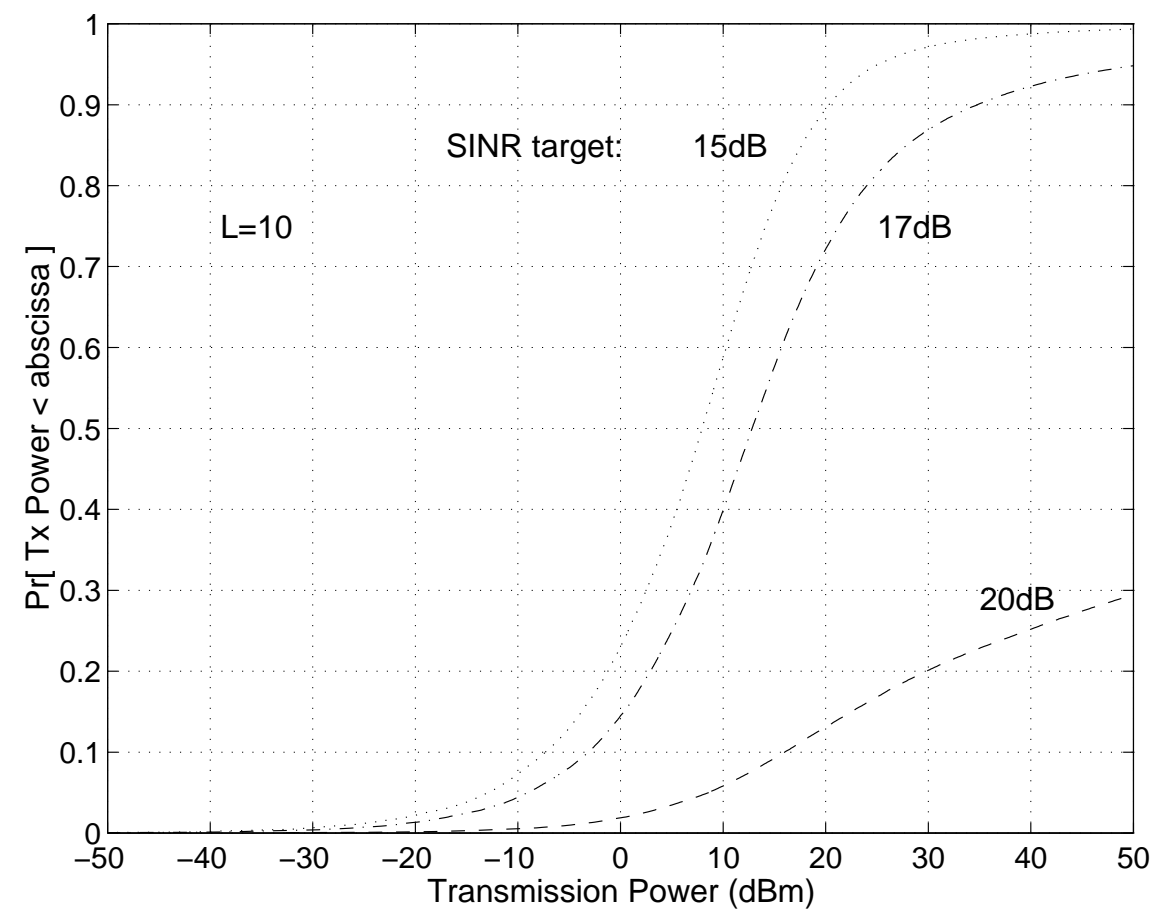

Table 1. Comparison Between the Kalman-Filter Method and the Delta-Modulation Method.

\begin{tabular}{|c|c|r|r|r|}
\hline \multirow{2}{*}{$\begin{array}{c}\text { Average Message } \\
\text { Length (Slots) }\end{array}$} & \multirow{2}{*}{ Method } & \multicolumn{3}{|c|}{ SINR Percentile (dB) } \\
\cline { 3 - 5 } & & $90 \%$ & $95 \%$ & $99 \%$ \\
\hline 3 & Kalman & 13.40 & 11.01 & 4.92 \\
3 & Delta & 11.96 & 8.90 & 2.94 \\
\hline 5 & Kalman & 14.37 & 12.33 & 6.28 \\
5 & Delta & 12.44 & 9.36 & 3.14 \\
\hline 10 & Kalman & 15.29 & 13.54 & 7.52 \\
10 & Delta & 12.99 & 9.88 & 3.31 \\
\hline 20 & Kalman & 16.02 & 14.53 & 8.38 \\
20 & Delta & 13.82 & 10.61 & 3.66 \\
\hline 40 & Kalman & 16.50 & 15.38 & 9.52 \\
40 & Delta & 15.19 & 12.15 & 5.39 \\
\hline
\end{tabular}


Table 2. Packet Error Rate for a Fading Channel Using 8-PSK Modulation.

\begin{tabular}{|c|c|}
\hline SINR $(\mathrm{dB})$ & Packet Error Rate \\
\hline 0 & 1.0 \\
5 & 0.8 \\
10 & 0.25 \\
15 & 0.024 \\
19 & 0.001 \\
\hline
\end{tabular}

Table 3. Comparison of Throughput and Packet Error Rate.

\begin{tabular}{|c|c|c|c|}
\hline $\begin{array}{c}\text { Avg. Message } \\
\text { Length (Slots) }\end{array}$ & Method & $\begin{array}{c}\text { Throughput } \\
(\text { Kbps })\end{array}$ & $\begin{array}{c}\text { Packet Error } \\
\text { Rate (PER) }\end{array}$ \\
\hline- & No Control & 21.14 & 0.073 \\
\hline 3 & Kalman & 21.71 & 0.048 \\
3 & Simple & 21.24 & 0.068 \\
\hline 5 & Kalman & 21.91 & 0.039 \\
5 & Simple & 21.42 & 0.060 \\
\hline 10 & Kalman & 21.96 & 0.037 \\
10 & Simple & 21.53 & 0.056 \\
\hline 20 & Kalman & 22.16 & 0.028 \\
\hline
\end{tabular}


Figure 5. Throughput Improvement of the Kalman-Filter Method.

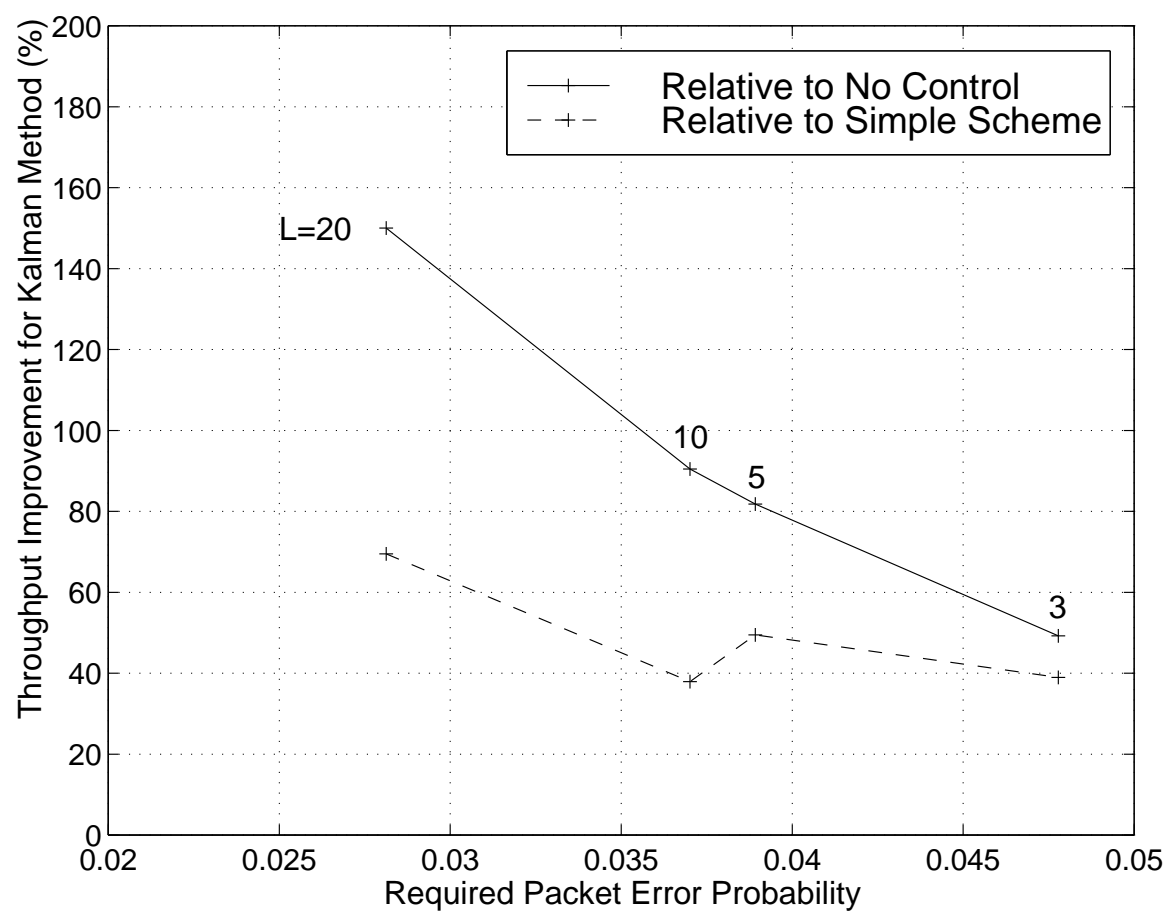

